\title{
Study on Reverse Reconstruction Method of Vehicle Group Situation in Urban Road Network Based on Driver-Vehicle Feature Evolution
}

\author{
Xiaoyuan Wang, ${ }^{1,2}$ Jianqiang Wang, ${ }^{2}$ Zhenxue Liu, ${ }^{1}$ Yaqi Liu, ${ }^{1}$ and Jingheng Wang ${ }^{3}$ \\ ${ }^{1}$ School of Transportation and Vehicle Engineering, Shandong University of Technology, Zibo 255049, China \\ ${ }^{2}$ State Key Laboratory of Automotive Safety and Energy, Tsinghua University, Beijing 100084, China \\ ${ }^{3}$ Shandong Zibo Experimental High School, Zibo 255000, China \\ Correspondence should be addressed to Xiaoyuan Wang; wangxiaoyuan@sdut.edu.cn
}

Received 28 December 2016; Accepted 26 January 2017; Published 20 February 2017

Academic Editor: Nicolas Hudon

Copyright (C) 2017 Xiaoyuan Wang et al. This is an open access article distributed under the Creative Commons Attribution License, which permits unrestricted use, distribution, and reproduction in any medium, provided the original work is properly cited.

Vehicle group situation is the status and situation of dynamic permutation which is composed of target vehicle and neighboring traffic entities. It is a concept which is frequently involved in the research of traffic flow theory, especially the active vehicle security. Studying vehicle group situation in depth is of great significance for traffic safety. Three-lane condition was taken as an example; the characteristics of target vehicle and its neighboring vehicles were synthetically considered to restructure the vehicle group situation in this paper. The Gamma distribution theory was used to identify the vehicle group situation when target vehicle arrived at the end of the study area. From the perspective of driver-vehicle feature evolution, the reverse reconstruction method of vehicle group situation in the urban road network was proposed. Results of actual driving, virtual driving, and simulation experiments showed that the model established in this paper was reasonable and feasible.

\section{Introduction}

The vehicle group situation which is composed of the dynamic traffic entities is the core content of traffic situation [1]. Studying the reduction method of vehicle group situation accurately is of great significance for the research on traffic flow theory and active vehicle security. The assessment and prediction of traffic situation was considered in most of previous studies. Käfer et al. [2] studied the recognition of situations involving two vehicles at intersections. The motion trajectories of the two vehicles were estimated ahead of 24 seconds based on a motion database, and the predicted trajectories were used to identify and predict traffic situation. The GPS data obtained from actual driving experiments was used to verify the model and the result showed that the method could recognize the situation correctly within 1.5-3 seconds before the vehicle reached the center of intersection. Meyer-Delius et al. [3] proposed a probabilistic approach to simulate and recognize traffic situation. The traffic situation was defined as a series of states that have practical significance, and Hidden Markov Model was used to obtain the probability distribution and the evolution law of traffic situation for typical traffic scenes. The behavior characteristics of drivers in complex and varied driving scenes were not fully considered and the systematic research on vehicle group situation was deficient in previous studies. The vehicle group situation in the two-lane case was divided and simplified by Zhang et al. [4] according to the spatial distribution of the target vehicle and its neighboring vehicles. The simplified vehicle group situation included eight states. On this basis, Wang et al. did many further studies; the division and simplification method of vehicle group situation in the three-lane case was studied. However, only the location distribution of vehicles was considered in the processes of division and simplification for vehicle group situation mentioned above, and the effects of the following vehicle, vehicle type, and driver characteristics, such as driving propensity, were ignored. Wang et al. considered the characteristics of vehicle type, speed, and location in the interest-sensitive area to build the mathematical expression of vehicle group 
situation. However, the impact of the driver characteristics on the vehicle group situation was not considered.

The appearance and development of GPS provided a new method for data acquisition in many fields. Meanwhile, the problems especially the issue of privacy disclosure are obviously increasing with the pervasive application of GPS. The cases of being deceived, property damage, and even casualties occurred repeatedly due to the privacy disclosure of position and trajectory. Hence privacy protection has become the focus of the whole society. Many scholars in the field of transportation engineering have attached great importance to the research on privacy protection constantly. Sun and Ban [5] proposed a method to reconstruct the vehicle trajectories for signalized intersections based on short-term trajectories of sample vehicle obtained from mobile traffic sensors. The previous studies focused on the knowledge extraction of city-scale traffic; the knowledge extraction of the fine-grained urban traffic was ignored. On this basis, Ban and Gruteser [6] summarized and analyzed the knowledge extraction of fine-grained urban traffic. The traffic modeling technology and privacy protection algorithm based on code signing were combined to extract the knowledge of finegrained urban traffic, and the performance of traffic signal control system was evaluated. Researchers pointed out that the privacy protection in intersection of urban road has been studied, and the privacy protection model for arterial roads (including some intersections) and arterial network would be the study emphasis in further research. The privacy protection technologies adapted to different cities should be developed.

At present, the combination of privacy protection and traffic application was rarely considered in the field of transportation engineering. Due to the low penetration rate of GPS and the growing awareness of privacy protection, it is difficult for researchers to collect the driving information of all vehicles. In addition, it would be difficult to process a large amount of data even if the driving information of all vehicles could be obtained. Therefore, studying the related issues with less information is of great significance; it benefits the privacy protection and solving traffic problems. In view of this, the three-lane scene was taken as an example, and the reduction method of vehicle group situation under the premise of the driver's privacy protection was studied in depth in this paper. The type, relative distance, and relative speed of the vehicles in the interest-sensitive area (including target vehicle) and the driver's propensity of target vehicle were used to divide vehicle group situation. And the reverse deduction method of vehicle group situation in urban road network using trajectory data of target vehicle was established based on the evolution of driver-vehicle feature. Finally, the experiments of actual driving, virtual driving, and simulation were designed to verify the method. Validation results showed that the proposed method could achieve the accuracy rate of $79 \%$ when penetration rate was $20 \%$. Under the premise of privacy protection for drivers, the research of reverse reconstruction method of vehicle group situation based on driver-vehicle feature evolution can not only improve the intelligent command system, but also lay

\begin{tabular}{ccc}
\multicolumn{1}{c}{ Driving direction } \\
\hline Right lane & Right front & Right rear \\
Middle lane & Front & Target \\
vehicle & Rear \\
Left lane & Left front & Left rear \\
\hline
\end{tabular}

(a)

\begin{tabular}{lll} 
& \\
Dight lane & Driving direction \\
\hline Middle lane & Feft lane & Target \\
vehicle & Rear \\
\hline
\end{tabular}

(b)

\begin{tabular}{|c|c|c|c|}
\hline \multicolumn{4}{|c|}{$\begin{array}{c}\text { Driving direction } \\
\text { Drion }\end{array}$} \\
\hline Right lane & Front & $\begin{array}{c}\text { Target } \\
\text { vehicle }\end{array}$ & Rear \\
\hline $\begin{array}{l}\text { Middle lane } \\
\text { Left lane }\end{array}$ & Left front & 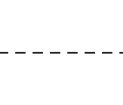 & Left rear - - - - \\
\hline
\end{tabular}

(c)

FIGURE 1: Division of interest-sensitive area.

a foundation for study and improvement of basic traffic flow model.

\section{Related Work}

2.1. Division of Vehicle Group Situation. In three-lane scene, the front bumper of target vehicle was taken as the dividing line, and 3 types of the interest-sensitive area when the target vehicle was in the middle lane, left lane, and right lane were shown as in Figures 1(a), 1(b), and 1(c).

The type, relative distance, and relative speed of the vehicles in the interest-sensitive area (including target vehicle) and the driver's propensity of target vehicle were used to divide vehicle group situation. The concept of "force" in physics was used to describe the effect of vehicles in each subarea on the target vehicle. The set of "force" (divided into repulsion and attraction force) was used to abstractly represent the vehicle group situation where target vehicle located. The calculation method of force was referred to in literature [7]. The divided vehicle group situations were shown as Figure 2 , where $Z_{1}, Z_{2}, \ldots, Z_{16}$, respectively, represented 16 kinds of vehicle group situations in three-lane scene. The symbol “+” represented attraction, and “-” represented repulsion.

\subsection{Recognition Method of Vehicle Group Situation Based on} Gamma Distribution. The time headway of vehicles reaching a cross section on the road can be described with Gamma distribution [8-10]. It is assumed that the time headway of vehicles arriving at the end of the road obeys the Gamma 


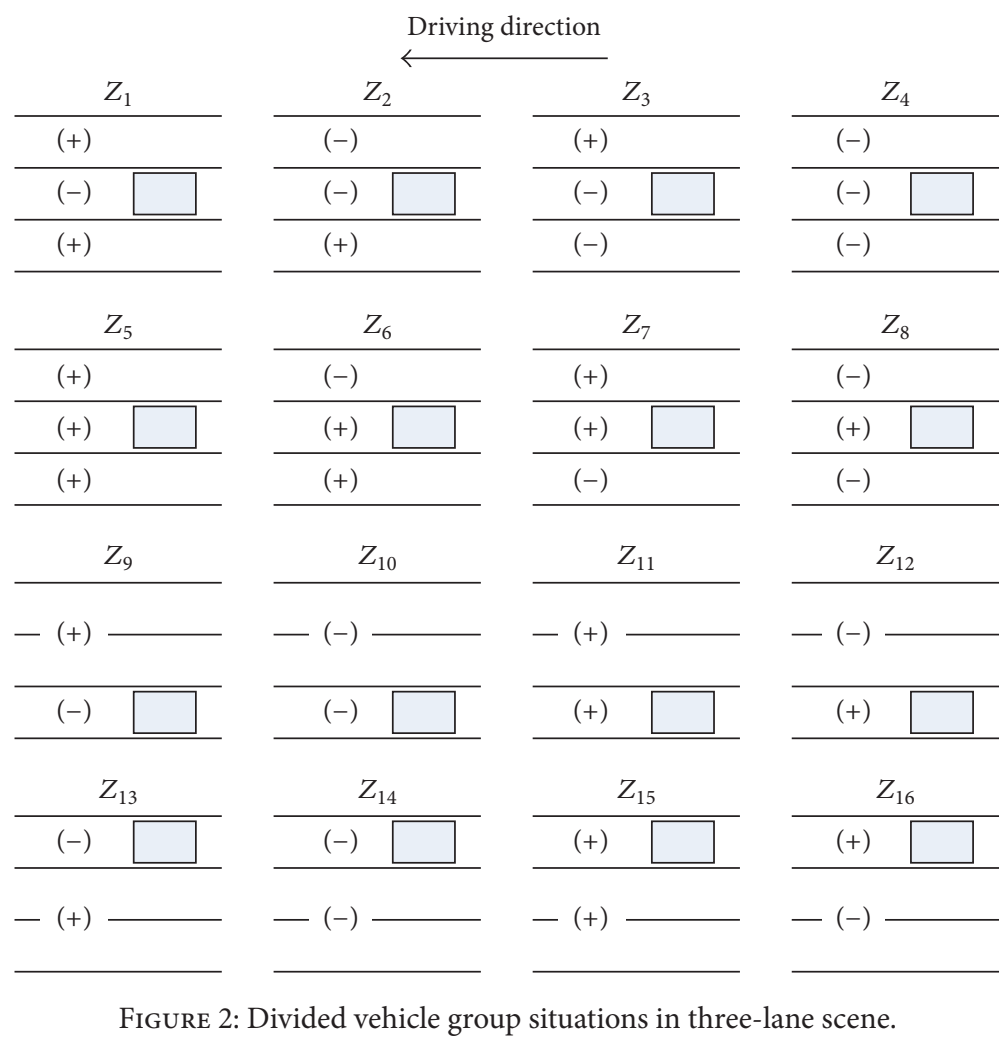

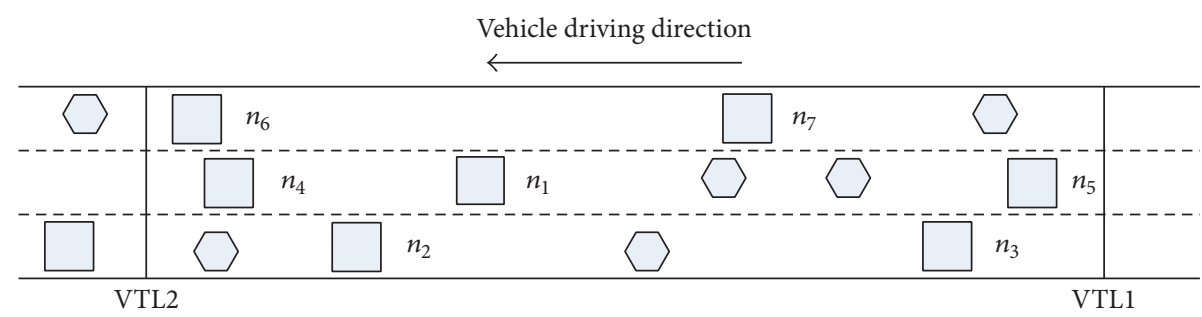

Experimental vehicle

Nonexperimental vehicle

FIGURE 3: Position distribution of the target vehicle and its neighboring vehicles.

distribution in this paper. The probability density function of Gamma distribution was shown as follows:

$$
f(x)=x^{k-1} \frac{e^{-x / \theta}}{\Gamma(k) \theta^{k}} .
$$

In formula (1), $k$ was shape parameter, $\theta$ was scale parameter, and $x \sim \Gamma(k, \theta)$. The corresponding parameters $k$ and $\theta$ could be obtained according to the historical data. Then the optimum mode of time headway could be obtained [10]:

$$
\text { mode }=(k-1) \theta \text {. }
$$

The location distribution of the target vehicle and its neighboring vehicles was shown in Figure 3.
Recognition steps of vehicle group situation when the target vehicle located at the end of the study area are as follows:

(1) Obtain the arrival time at VTL1 and VTL2 of target vehicle and its neighboring vehicles in different subareas when the target vehicle located at VTL2. The arrival times at VTL1 and VTL2 of target vehicle $n_{1}$, left front vehicle $n_{2}$, left rear vehicle $n_{3}$, front vehicle $n_{4}$, rear vehicle $n_{5}$, right front vehicle $n_{6}$, and right rear vehicle $n_{7}$ were marked as $t_{1}, t_{2}, t_{3}, t_{4}, t_{5}, t_{6}, t_{7}$ and $t_{1}^{\prime}, t_{2}^{\prime}, t_{3}^{\prime}, t_{4}^{\prime}, t_{5}^{\prime}, t_{6}^{\prime}, t_{7}^{\prime}$, respectively. According to the time information, the following work should be done, respectively:

(a) Calculate the travel time of experimental vehicles $T_{i}=t_{i}-t_{i}^{\prime}, i=1,2, \ldots, 7$, and then 


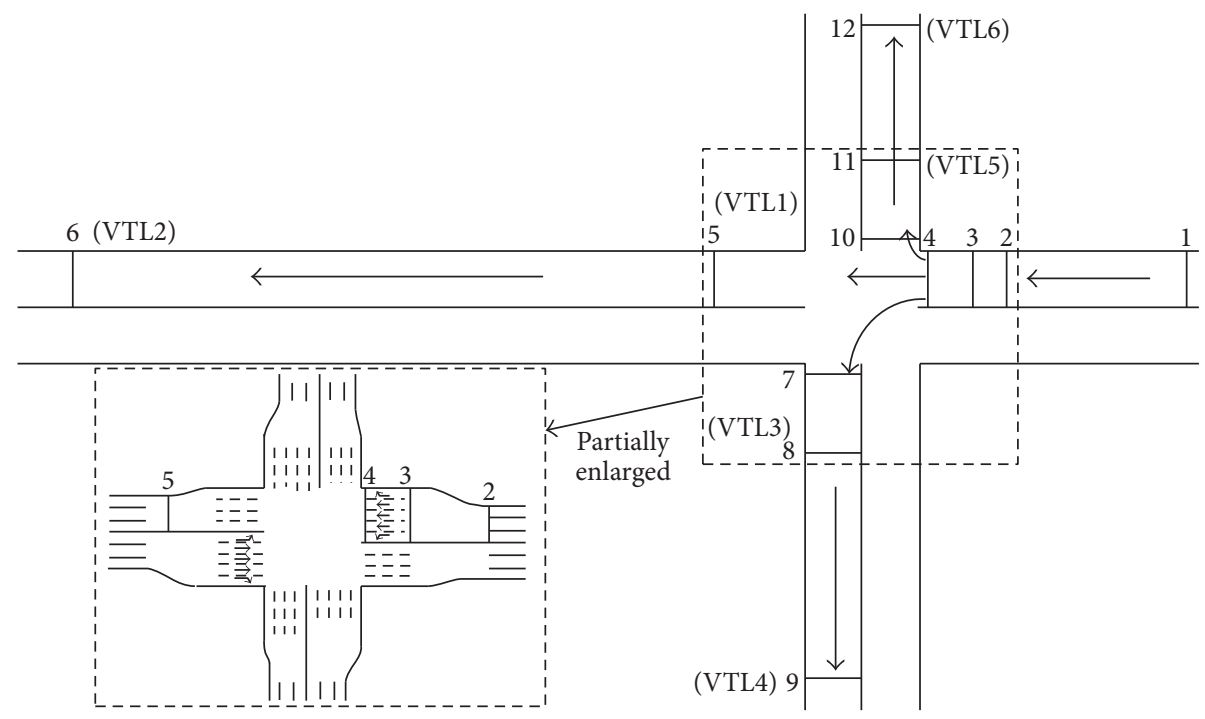

FIGURE 4: Schematic diagram of three-lane scene.

recognize the type and driver's propensity of target vehicle based on Bayesian decision tree dynamic recognition model [11].

(b) Calculate the difference of arrival time at VTL2 between the target vehicle and other experimental vehicles: $\Delta t_{i 1}=\left|t_{\mathrm{i}}^{\prime}-t_{1}^{\prime}\right|$. Calculate the difference of arrival time at VTL2 between experimental vehicle $n_{2}$ and $n_{3}: \Delta t_{23}=\left|t_{3}^{\prime}-t_{2}^{\prime}\right|$. Calculate the difference of arrival time at VTL2 between experimental vehicle $n_{6}$ and $n_{7}, \Delta t_{67}=$ $\left|t_{7}^{\prime}-t_{6}^{\prime}\right|$.

(c) Deduce the number of nonexperimental vehicles in the interest-sensitive area according to $\Delta t_{23}, \Delta t_{41}, \Delta t_{51}$, and $\Delta t_{67}$ and the optimum mode of time headway.

(d) Calculate the average speed of each experimental vehicle $v_{i}, v_{i}=L / T_{i}(i=1,2, \ldots, 7)$, where $L$ represented the distance between VTL1 and VTL2. $v_{2}, v_{3}, \ldots, v_{7}$ were used to approximately express the speed of left front vehicle, left rear vehicle, front vehicle, rear vehicle, right front vehicle, and right rear vehicle. The relative speed of the target vehicle and its neighboring vehicles could be estimated according to the average speed of the target vehicle.

(2) The nearest experimental vehicle from the target vehicle was taken as the standard; assume that various types of vehicles on the road obey uniform distribution in each lane and the types of neighboring vehicles were estimated according to the proportion of different types of vehicles on the road.

(3) Consider the type, position, and speed of neighboring vehicles and driver's propensity of target vehicle when the target vehicle arrived at VTL2; the force of each lane on the target vehicle could be obtained to recognize vehicle group situation at the end of the road.

\section{Methodology}

Hidden Markov Model (HMM) [12] is a time series statistical analysis model which consists of a Markov chain and a general stochastic process. The Markov chain is used to describe the evolution of driver-vehicle feature and the general stochastic process is used to describe the statistical characteristics of the observed output value for vehicle group situation.

The three-lane scene was taken as an example, and the reconstruction method of vehicle group situation was illustrated in this paper (shown as in Figure 4). The vehicle travel characteristic at the intersection was different from that on the road due to the traffic signal control. The transition laws of vehicle group situation were bound to be different when the vehicle ran in different areas. Therefore, the urban road network was divided into different areas with the dividing line based on a great deal of observation data. The transition laws of vehicle group situation in different areas were studied separately and the transition laws for the entire road network could be revealed ultimately.

In Figure 4, dividing line 1 was set on the ordinary road section of the intersection upstream without the influence of traffic signal. Dividing line 2 was set at the entrance of widening section and dividing line 3 was set at the exit of widening section upstream of the intersection. Dividing line 4 was arranged at the position of the stop line. Dividing lines 5,8 , and 11 were arranged at the exit of widening section downstream of the intersection. Dividing lines 6, 9 and 12 were arranged on the ordinary road section downstream of the intersection. Dividing lines 7 and 10 were arranged behind the crosswalks downstream of the intersection. The Virtual Trip Lines were set at the dividing lines 5, 6, 8, 9, 11, and 12 
which were represented by VTL1, VTL2, VTL3, VTL4, VTL5, and VTL6, respectively [13].

3.1. Reconstruction Method of Vehicle Group Situation on the Road Section. The road section between VTL1 (dividing line 5) and VTL2 (dividing line 6) in Figure 3 was taken as an example to illustrate the steps of vehicle group situation reconstruction:

(1) The travel time of experimental vehicle between dividing lines 5 and 6 was estimated according to the arrival time at VTL1 and VTL2. Then the vehicle group situation was recognized with Gamma distribution when the target vehicle located at dividing line 6.

(2) Assume that the transition of vehicle group situation and driver-vehicle feature had Markov property and the vehicle group situation at time $t$ was affected by the corresponding driver-vehicle feature. On account of the fact that the vehicle type was not changed when driving, only the driving propensity was changed; thus the driver-vehicle feature transition represented the evolution of driving propensity here. The influences of driver-vehicle feature on vehicle group situation at different times were shown in Figure 5.

Hidden Markov Model was used to reveal the transition mechanism of vehicle group situation adapting to the timevarying characteristic of driver-vehicle feature in this paper. The HMM was represented by $\lambda=(F, Z, \pi, A, B)$. The parameters were described as follows:

(1) The small car and its driver's propensity were taken as an example. Because the vehicle type did not change with the time, the set of driver-vehicle features (small car) was marked as $F=\left\{F_{1}, F_{2}, F_{3}\right\}$. And the state of Markov chain at time $t$ was marked as $e_{t}$.

(2) The set of vehicle group situation was denoted as $Z=$ $\left\{Z_{1}, Z_{2}, \ldots, Z_{16}\right\}$. The state of vehicle group situation at time $t$ was marked as $x_{t}$.

(3) The state probability distribution matrix of initial driver-vehicle feature was marked as $\pi=\left\{\pi_{1}, \pi_{2}, \pi_{3}\right\}$. The probability of state $F_{i}$ when $t=1$ was represented by $\pi_{i}=P\left(e_{1}=F_{i}\right), i=1,2,3$.

(4) The transition probability matrix of driver-vehicle feature was marked as $A=\left(a_{i j}\right)_{3 \times 3}, a_{i j}=P\left(e_{t+1}=\right.$ $\left.F_{j} \mid e_{t}=F_{i}\right), i, j=1,2,3 . a_{i j}$ represented the probability that driver-vehicle feature changed from state $F_{i}$ at time $t$ to state $F_{j}$ at time $t+1$.

(5) The probability distribution matrix of vehicle group situation under the given driver-vehicle feature was

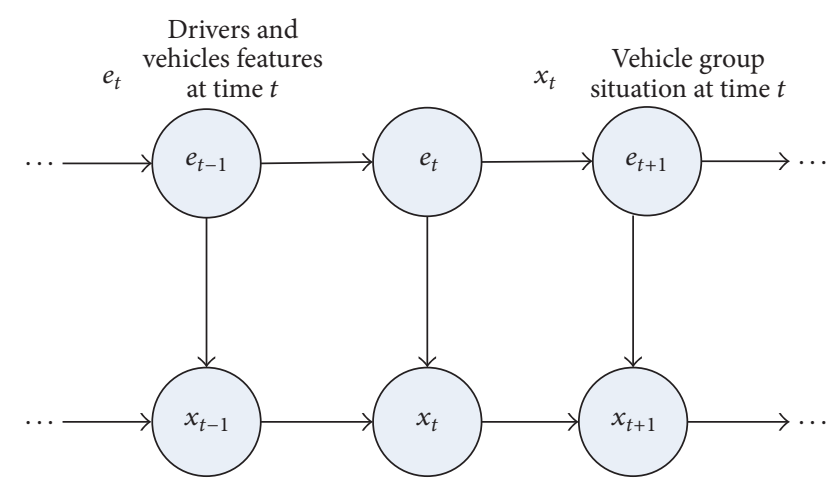

FIGURE 5: Influences of driver-vehicle feature on vehicle group situation at different times.

marked as $B=\left(b_{j}\left(u_{k}\right)\right)_{3 \times 16}, b_{j}\left(u_{k}\right)=P\left(x_{k}=u_{k} \mid\right.$ $\left.e_{k}=F_{j}\right), 1 \leq j \leq 3,1 \leq k \leq 16 . b_{j}\left(u_{k}\right)$ represented the probability of vehicle group situation $x_{k}=u_{k}$ when driver-vehicle feature was $F_{j}$ at time $k$, where $u_{k}$ was a value of $Z=\left\{Z_{1}, Z_{2}, \ldots, Z_{16}\right\}$.

The Baum-Welch estimation algorithm was used to select and optimize parameters for $\lambda=(F, Z, \pi, A, B)[14,15]$.

Firstly, the forward variable $\alpha_{t}(i)$ and backward variable $\beta_{t}(i)$ were given as follows:

$$
\alpha_{t}(i)=P\left(u_{1}, u_{2}, \ldots, u_{t} ; e_{t}=F_{i}\right) \quad 1 \leq i \leq 16 .
$$

The forward variable $a_{t}(i)$ represented the probability when the driver-vehicle feature was $F_{i}$ at time $t$ and the observed vehicle group situation $x_{k}(k=1,2, \ldots, t)$ was $u_{k}(k=1,2, \ldots, t)$ at time $k(k=1,2, \ldots, t)$, where $\alpha_{1}(i)=\pi_{i} b_{i}\left(u_{1}\right)$ represented the probability when vehicle group situation sequence $x_{1}=u_{1}$ and driver-vehicle feature was $F_{i}$ at the initial moment $t=1$.

$$
\begin{aligned}
\beta_{t}(i)=P\left(u_{t+1}, u_{t+2}, \ldots, u_{L} \mid e_{t}\right. & \left.=F_{i}\right) \\
1 & \leq i \leq 16,1 \leq t \leq L-1 .
\end{aligned}
$$

The backward variable $\beta_{t}(i)$ represented the probability that observed vehicle group situation $x_{k}(k=t+1, t+2, \ldots, L)$ was $u_{k}(k=t+1, t+2, \ldots, L)$ at time $k(k=t+1, t+$ $2, \ldots, L)$ when the driver-vehicle feature was $F_{i}$ at time $t$. Set the initial value $\beta_{L}(i)=1$, which represented the probability of the termination state of observed vehicle group situation sequence $y_{L}=u_{L}$ at the time $t=L$ when the driver-vehicle feature was $F_{i}$, and that was a certain event.

The recurrence formula of $\alpha_{t}(i)$ and $\beta_{t}(i)$ could be obtained (shown as (5)) according to the influences of drivervehicle feature on vehicle group situation at different times:

$$
\begin{aligned}
\alpha_{t+1}(j) & =P\left(u_{1}, u_{2}, \ldots, u_{t+1} ; e_{t+1}=F_{j}\right)=P\left(u_{t+1} \mid u_{1}, u_{2}, \ldots, u_{t} ; e_{t+1}=F_{j}\right) P\left(u_{1}, u_{2}, \ldots, u_{t} ; e_{t+1}=F_{j}\right) \\
& =P\left(u_{t+1} \mid u_{t} ; e_{t+1}=F_{j}\right) \sum_{i=1}^{3} P\left(u_{1}, u_{2}, \ldots, u_{t} ; e_{t}=F_{i}\right) P\left(e_{t+1}=F_{j} \mid e_{t}=F_{i}\right)
\end{aligned}
$$




$$
\begin{aligned}
& =\left[\sum_{i=1}^{3} \alpha_{t}(i) a_{i j}\right] \frac{P\left(u_{t}, u_{t+1} ; e_{t+1}=F_{j}\right)}{P\left(u_{t} ; e_{t+1}=F_{j}\right)} \\
& =\left[\sum_{i=1}^{3} \alpha_{t}(i) a_{i j}\right] \frac{P\left(e_{t+1}=F_{j}\right) P\left(u_{t+1} \mid e_{t+1}=F_{j}\right) P\left(u_{t} \mid u_{t+1} ; e_{t+1}=F_{j}\right)}{P\left(u_{t}\right) P\left(e_{t+1}=F_{j}\right)}=\left[\sum_{i=1}^{3} \alpha_{t}(i) a_{i j}\right] \frac{b_{j}\left(u_{t+1}\right) P\left(u_{t} \mid u_{t+1}\right)}{P\left(u_{t}\right)} \\
& 1 \leq j \leq 3,1 \leq t \leq L-1,
\end{aligned}
$$

$$
\begin{aligned}
\beta_{t}(i) & =P\left(u_{t+1}, u_{t+2}, \ldots, u_{L} \mid e_{t}=F_{i}\right)=\sum_{j=1}^{3} P\left(u_{t+1}, u_{t+2}, \ldots, u_{L} ; e_{t+1}=F_{j} \mid e_{t}=F_{i}\right) \\
& =\sum_{j=1}^{3} P\left(e_{t+1}=F_{j} \mid e_{t}=F_{i}\right) P\left(u_{t+1}, u_{t+2}, \ldots, u_{L} \mid e_{t}=F_{i}, e_{t+1}=F_{j}\right) \\
& =\sum_{j=1}^{3} P\left(e_{t+1}=F_{j} \mid e_{t}=F_{i}\right) P\left(u_{t+1} \mid u_{t+2}, \ldots, u_{L} ; e_{t}=F_{i}, e_{t+1}=F_{j}\right) P\left(u_{t+2}, u_{t+3}, \ldots, u_{L} \mid e_{t}=F_{i}, e_{t+1}=F_{j}\right) \\
& =\sum_{j=1}^{3} a_{i j} \frac{P\left(e_{t+1}=F_{j}\right) P\left(u_{t+1} \mid e_{t+1}=F_{j}\right) P\left(u_{t+2} \mid u_{t+1} ; e_{t+1}=F_{j}\right)}{P\left(u_{t+2}\right) P\left(e_{t+1}=F_{j}\right)} \beta_{t+1}(j) \\
& =\sum_{j=1}^{3} a_{i j} b_{j}\left(u_{t+1}\right) \frac{P\left(u_{t+2} \mid u_{t+1}\right)}{P\left(u_{t+2}\right)} \beta_{t+1}(j) \quad 1 \leq j \leq 3,1 \leq t \leq L-1 .
\end{aligned}
$$

$\alpha_{t}(i)$ was used to deduce the partial sequence $u_{1}, u_{2}, \ldots, u_{t}$ of observation sequence $u_{1}, u_{2}, \ldots, u_{L} . \beta_{t}(i)$ was used to deduce the other part of the sequence $u_{t+1}, u_{t+2}, \ldots, u_{L}$. Then the probability of the whole state sequence $P\left(u_{1}, u_{2}, \ldots, u_{L}\right)$ could be obtained:

$$
\begin{aligned}
& P\left(u_{1}, u_{2}, \ldots, u_{L}\right)=\sum_{i=1}^{3} P\left(u_{1}, u_{2}, \ldots, u_{L} ; e_{t}=F_{i}\right) \\
& \quad=\sum_{i=1}^{3} P\left(u_{1}, u_{2}, \ldots, u_{t} ; e_{t}=F_{i}\right) \\
& \quad \cdot P\left(u_{t+1}, u_{t+2}, \ldots, u_{L} \mid u_{1}, u_{2}, \ldots, u_{t}, e_{t}=F_{i}\right)
\end{aligned}
$$

$$
\begin{aligned}
& =\sum_{i=1}^{3} \alpha_{t}(i) P\left(u_{t+1}, \ldots, u_{L} \mid u_{t} ; e_{t}=F_{i}\right)=\sum_{i=1}^{3} \alpha_{t}(i) \\
& \cdot \frac{P\left(u_{t}, \ldots, u_{L} ; e_{t}=F_{i}\right)}{P\left(u_{t}, e_{t}=F_{i}\right)}=\sum_{i=1}^{3} \alpha_{t}(i) \beta_{t}(i) \\
& \cdot \frac{P\left(u_{t+1} \mid u_{t}\right)}{P\left(u_{t+1}\right)} .
\end{aligned}
$$

$\alpha_{t}(i)$ and $\beta_{t}(i)$ were used to optimize the model. And the variables $\varepsilon_{t}(i, j)$ and $\gamma_{t}(i)$ were defined as

$$
\begin{aligned}
\varepsilon_{t}(i, j) & =P\left(e_{t}=F_{i}, e_{t+1}=F_{j} \mid u_{1}, u_{2}, \ldots, u_{t}, \ldots, u_{L}\right)=\frac{P\left(e_{t}=F_{i}, e_{t+1}=F_{j} ; u_{1}, \ldots, u_{t}, \ldots, u_{L}\right)}{P\left(u_{1}, \ldots, u_{t}, \ldots, u_{L}\right)} \\
& =\frac{P\left(e_{t}=F_{i}, e_{t+1}=F_{j} ; u_{1}, \ldots, u_{t}\right) P\left(u_{t+1}, \ldots, u_{L} \mid u_{1}, \ldots, u_{t} ; e_{t}=F_{i}, e_{t+1}=F_{j}\right)}{P\left(u_{1}, \ldots, u_{t}, \ldots, u_{L}\right)} \\
& =\frac{P\left(e_{t}=F_{i} ; u_{1}, u_{2}, \ldots, u_{t}\right) P\left(e_{t+1}=F_{j} \mid e_{t}=F_{i} ; u_{1}, \ldots, u_{t}\right) P\left(u_{t+1}, \ldots, u_{L} \mid u_{t} ; e_{t}=F_{i}, e_{t+1}=F_{j}\right)}{P\left(u_{1}, \ldots, u_{t}, \ldots, u_{L}\right)} \\
& =\frac{\alpha_{t}(i) a_{i j} b_{j}\left(u_{t+1}\right) \beta_{t}(i)}{\sum_{i=1}^{3} \alpha_{t}(i) \beta_{t}(i) P\left(u_{t+1}\right)} \quad 1 \leq t \leq L-1,
\end{aligned}
$$




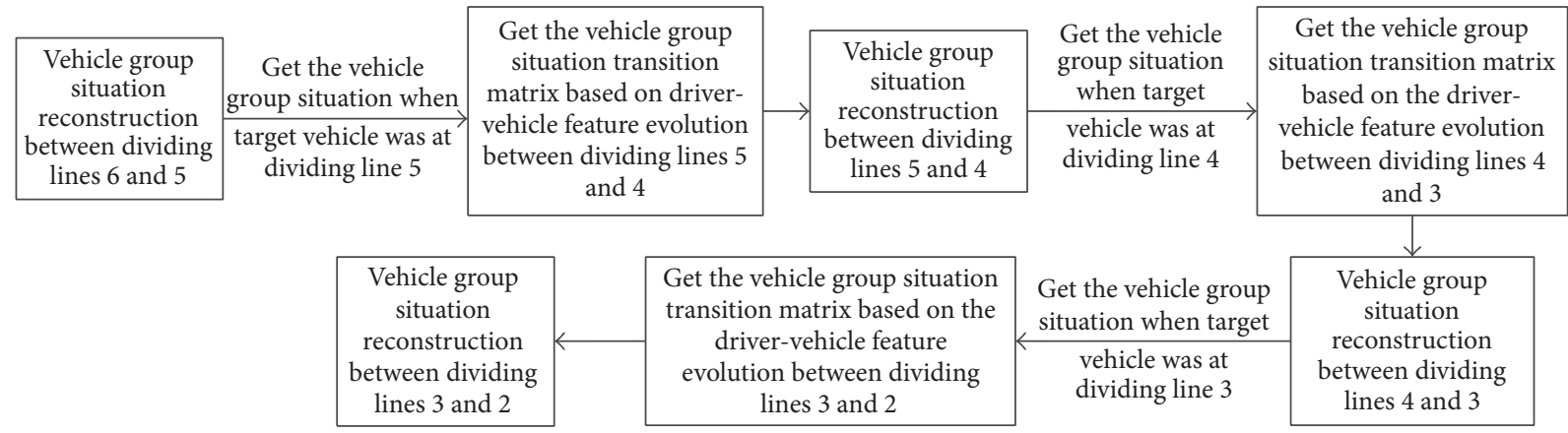

FIGURE 6: Reconstruction process of vehicle group situation for going straight at the intersection.

where $\varepsilon_{t}(i, j)$ represented the probability that the drivervehicle feature was $F_{i}$ at time $t$ and the driver-vehicle feature was $F_{j}$ at time $t+1$ when the given vehicle group situation sequence was $u_{1}, u_{2}, \ldots, u_{L}$ :

$$
\begin{aligned}
& \gamma_{t}(i)=P\left(e_{t}=F_{i} \mid u_{1}, \ldots, u_{t}, \ldots, u_{L}\right) \\
& =\frac{P\left(e_{t}=F_{i}, u_{1}, \ldots, u_{t}, \ldots, u_{L}\right)}{P\left(u_{1}, \ldots, u_{t}, \ldots, u_{L}\right)} \\
& =\frac{P\left(e_{t}=F_{i} ; u_{1}, \ldots, u_{t}\right) P\left(u_{t+1}, \ldots, u_{L} \mid u_{1}, \ldots, u_{t} ; e_{t}=F_{i}\right)}{P\left(u_{1}, \ldots, u_{t}, \ldots, u_{L}\right)} \\
& =\frac{\alpha_{t}(i) P\left(u_{t+1}, \ldots, u_{L} \mid u_{t} ; e_{t}=F_{i}\right)}{P\left(u_{1}, \ldots, u_{t}, \ldots, u_{L}\right)} \\
& =\frac{\alpha_{t}(i)}{P\left(u_{1}, \ldots, u_{L}\right)} \beta_{t}(i) \frac{P\left(u_{t+1} \mid u_{t}\right)}{P\left(u_{t+1}\right)}=\frac{\alpha_{t}(i) \beta_{t}(i)}{\sum_{i=1}^{3} \alpha_{t}(i) \beta_{t}(i)} \\
& 1 \leq t \leq L-1 .
\end{aligned}
$$

$\gamma_{t}(i)$ represented the probability that the driver-vehicle feature was $F_{i}$ at time $t$ under the given vehicle group situation sequence $u_{1}, u_{2}, \ldots, u_{L}$.

The optimized transition probability formula of drivervehicle feature could be obtained according to the derived parameters, shown as

$$
\begin{aligned}
\hat{a}_{i j} & =\frac{\sum_{t=1}^{L-1} P\left(e_{t}=F_{i}, e_{t+1}=F_{j} \mid u_{1}, \ldots, u_{L}\right)}{\sum_{t=1}^{L-1} P\left(e_{t}=F_{i} \mid u_{1}, \ldots, u_{L}\right)} \\
& =\frac{\sum_{t=1}^{L-1} \varepsilon_{t}(i, j)}{\sum_{t=1}^{L-1} \gamma_{t}(i)}=\frac{\sum_{t=1}^{L-1} a_{i j} b_{j}\left(u_{t+1}\right)}{\sum_{t=1}^{L-1} \sum_{j=1}^{3} a_{i j} b_{j}\left(u_{t+1}\right)} .
\end{aligned}
$$

The optimization algorithm for the probability distribution of vehicle group situation was as follows:

$$
\begin{aligned}
\widehat{b}_{j}\left(u_{k}\right) & =\frac{\sum_{t=1, y_{t}=u_{k}}^{L} P\left(e_{t}=F_{j} \mid u_{1}, \ldots, u_{L}\right)}{\sum_{t=1}^{L} P\left(e_{t}=F_{j} \mid u_{1}, \ldots, u_{L}\right)} \\
& =\frac{\sum_{t=1, y_{t}=u_{k}}^{L} \gamma_{t}(j)}{\sum_{t=1}^{L} \gamma_{t}(j)}=\frac{\sum_{t=1, y_{t}=u_{k}}^{L} \alpha_{t}(j) \beta_{t}(j)}{\sum_{t=1}^{L} \alpha_{t}(j) \beta_{t}(j)} .
\end{aligned}
$$

The optimization algorithm for the probability distribution of initial driver-vehicle feature $\pi_{i}$ was

$$
\widehat{\pi}_{i}=\gamma_{1}(i)=\frac{\alpha_{1}(i) \beta_{1}(i)}{\sum_{i=1}^{3} \alpha_{1}(i) \beta_{1}(i)} .
$$

The transition probability of vehicle group situation from time $t-1$ to time $t$ was denoted as $p_{i, j}(i, j=1,2,3, \ldots, 16)$. The reverse transition probability matrix of vehicle group situation could be obtained according to the probability theory, where vehicle group situation was $Z_{j}$ at time $t$, and vehicle group situation was $Z_{i}$ at time $t-1$. The reverse transition probability was denoted as $p_{j, i}(j, i=1,2,3, \ldots, 16)$. The derivation formula was shown as

$$
p_{j, i}=\frac{p\left(Z_{i}\right) \cdot p_{i, j}}{\sum_{k=1}^{16}\left[p\left(Z_{k}\right) \cdot p_{k, j}\right]}, \quad j, i=1,2,3, \ldots, 16 .
$$

In (12), $p\left(Z_{k}\right)$ represented the probability of vehicle group situation $Z_{k}, k=1,2,3, \ldots, 16$.

According to the above method, the vehicle group situation of the dividing lines 2-1, 9-8, and 12-11 could be reconstructed.

3.2. Reconstruction Method of Vehicle Group Situation for Going Straight. The number of straight-going lanes between dividing lines 5 and 4 was constant and the right-turning and left-turning vehicles had exclusive lanes; thus the impacts of the right-turning and left-turning vehicles on the vehicle which was going straight could be ignored. Therefore, the transition matrix construction method of vehicle group situation for the region between dividing lines 6 and 5 was also applicable to the section between dividing lines 5 and 4. Similarly, the vehicle group situation for going straight vehicles in the region between dividing lines 4 and 3 and 3 and 2 could be reconstructed. The reconstruction process of vehicle group situation for going straight at the intersection was shown as in Figure 6.

\subsection{Reconstruction Method of Vehicle Group Situation for} Turning Right and Turning Left. There were exclusive lanes in the region of the intersection for the right-turning and leftturning vehicles. For the convenience of study, the impacts of 


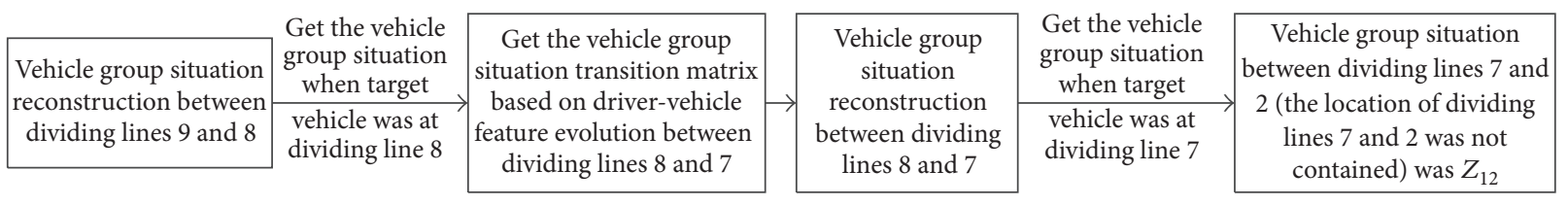

FiguRE 7: Reconstruction of vehicle group situation for left-turning vehicles.

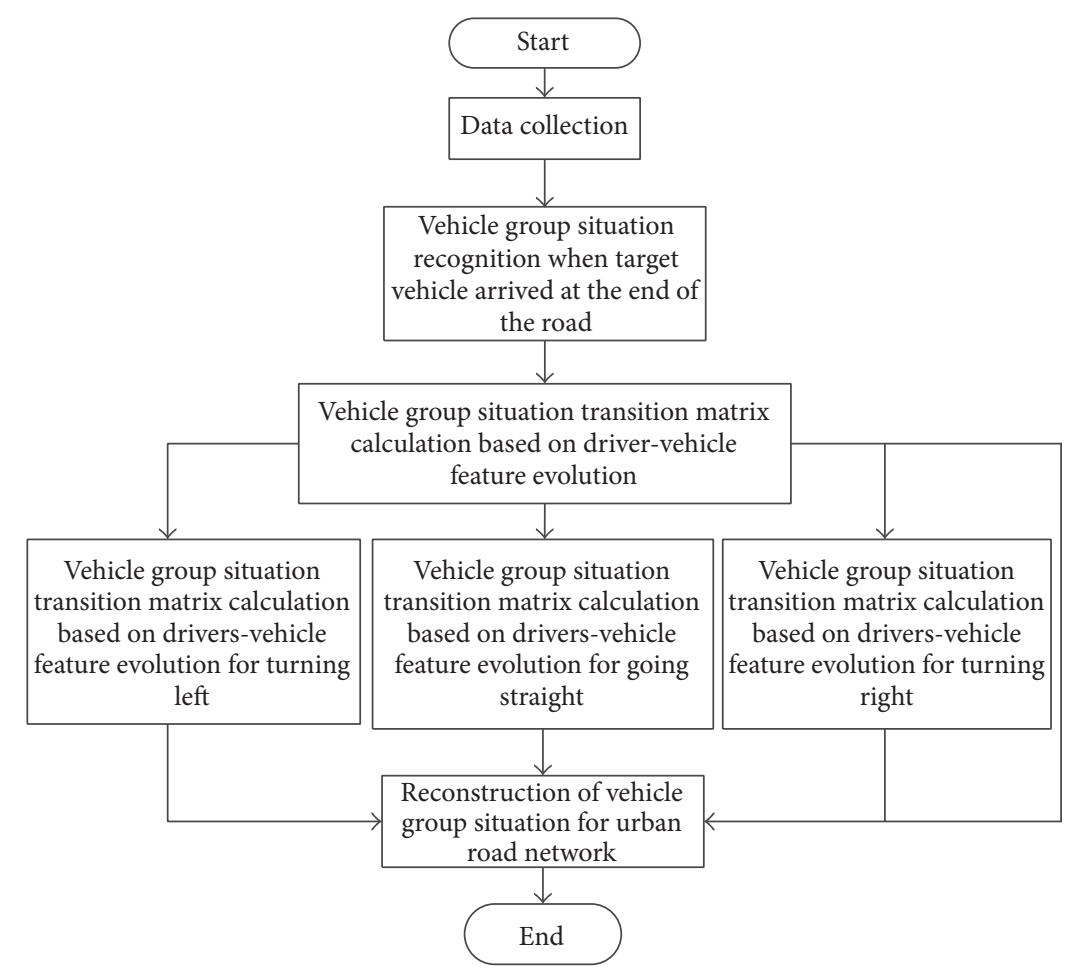

FIGURE 8: Reverse reconstruction process of vehicle group situation for urban road network.

vehicles running in other directions on the right-turning and left-turning vehicles were ignored. The impacts of the rightturning and left-turning vehicles on the vehicle which was going straight were ignored. For left-turning vehicle between dividing lines 7 and 4, 4 and 3, and 3 and 2, the impacts from the right-turning and going straight vehicles were ignored and it could only run in the exclusive lane; thus the vehicle group situation was regarded as $Z_{12}$. Similarly, the vehicle group situation for right-turning vehicles between dividing lines 10 and 4,4 and 3 , and 3 and 2 was regarded as $Z_{16}$.

The reconstruction of vehicle group situation for leftturning vehicles was taken as an example, and the reconstruction process of vehicle group situation was illustrated as in Figure 7.

3.4. Reconstruction Method of Vehicle Group Situation for Urban Road Network. Three-lane road network was composed of multiple regions, and one of the regions was shown as in Figure 3 (excepting the region between dividing lines 2 and 1). The vehicle group situation in the whole road network could be reconstructed according to the vehicle group situation transition information of each region. The reverse reconstruction process of vehicle group situation for urban road network was shown as in Figure 8.

\section{Experimental Design}

4.1. Experimental Equipment. The experimental data of vehicle driving was acquired in a city road environment through a dynamic driver-vehicle-environment information acquisition system (including BioHarness 3.0 portable physiological signal measurement instrument and SG299-GPS noncontact multifunction speedometer, as shown in Figure 9). In addition, many types of software were used in the experiment, such as PsyLAB software, SPSS19.0, and Corel Video Studio 10.0.

\subsection{Experimental Content}

(1) 50 drivers were organized to participate in a psychological questionnaire test, and the test method was referred to in literature $[1,4]$. The driving propensities of 50 drivers could be obtained.

(2) The time series data of influential factors on vehicle group situation (vehicle type, position, etc.) was collected in actual driving experiment.

(3) Experimental condition: the experiments were implemented at 8:30-10:30 in a normal working day with good weather and road condition. The traffic state was non-free flow. 


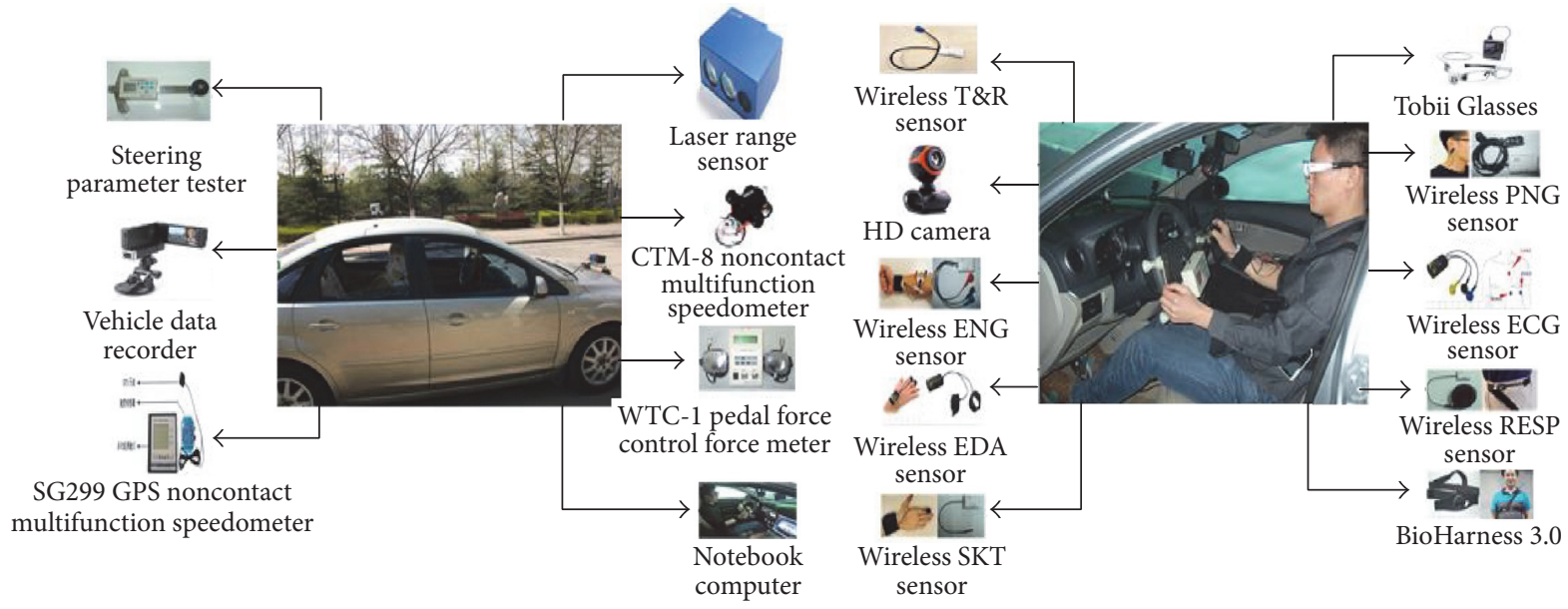

FIGURE 9: Dynamic driver-vehicle-environment information acquisition system.

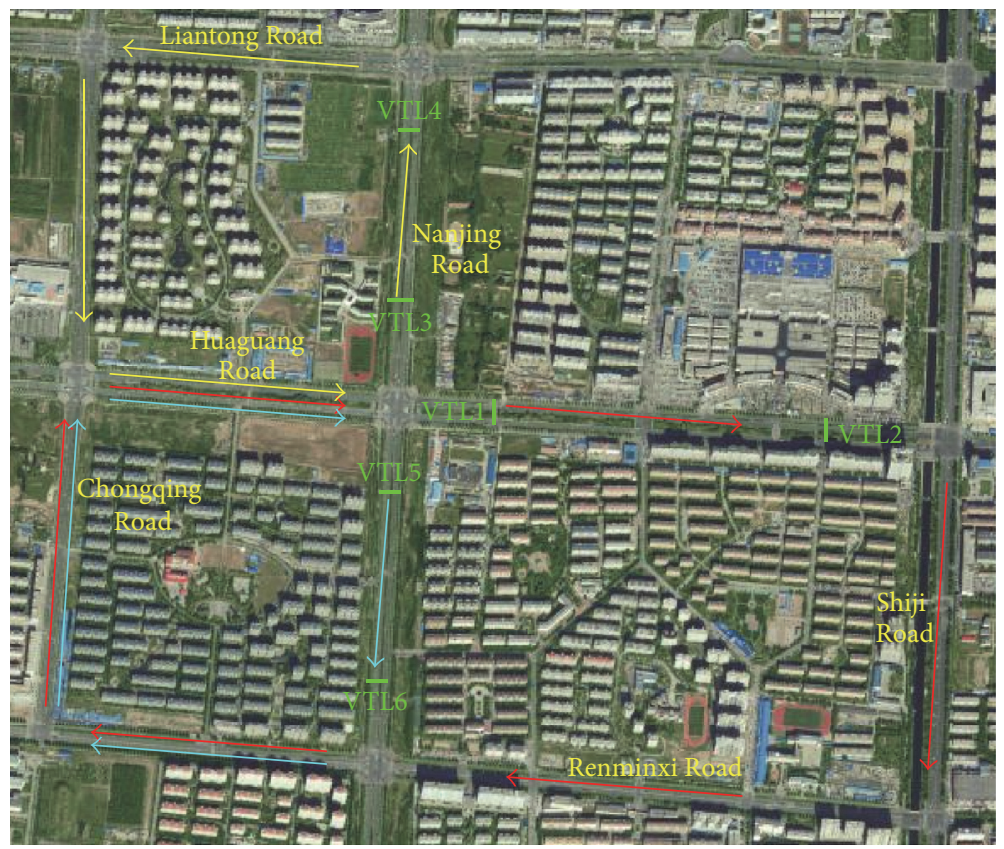

FIgURE 10: Experimental routes.

4.3. Experiment Location and Road Condition. Three experimental routes shown as in Figure 10 were selected to conduct the experiments. The experimental routes included Huaguang Road-Nanjing Road-Liantong Road-Chongqing Road (marked as yellow line), Huaguang Road-Nanjing Road-Renminxi Road-Chongqing Road (marked as blue line), and Huaguang Road-Shiji Road-Road-Renminxi RoadChongqing Road (marked as red route). 50 drivers were organized to drive experimental vehicles equipped with the dynamic information acquisition system on the experimental routes circularly. Drivers could change lanes freely according to their habits and preferences. Multiple groups of experimental data were collected from each driver. The information of arrival time was recorded when the vehicle arrived at VTL (green marker line). The high-definition camera, laser ranging, and so forth were used to record all the information of target vehicle, environment (inside and outside), and driver behavior for the further study.

\section{Result and Discussion}

5.1. Result. The reconstruction of the vehicle group situation under different penetration rates was studied in this paper. Limited to the space, only the reconstruction with penetration rate of $20 \%$ was taken as an example. Partial experimental data of 30 drivers was selected as sample data. The transition probability matrix of vehicle group situation was obtained according to the proposed method. And then the vehicle group situation in the experimental area was reconstructed. 
For the same type of vehicle, when a driver with different propensity drove the same type vehicle, the transition matrixes of the vehicle group situation were different. Similarly, for the same propensity, when a driver with the same propensity drove different type of vehicles, the transition matrixes of the vehicle group situation were different. Thus the effect of driver-vehicle feature evolution on the transition of vehicle group situation was complicated and multifarious. On account of the fact that vehicle type was not changed when driving, only the driving propensity was changed; thus the driver-vehicle feature transition represented the evolution of driving propensity for one piece of trajectory data. The transition of "small vehicle + common type" to "small vehicle + radical type" was taken as an example, and the transition matrix of the vehicle group situation in some regions was shown as follows, where $P(m-n)(1 \leq m \leq 12,1 \leq n \leq$ 12) represented the vehicle group situation transition matrix between dividing lines $m$ to $n$ :

$$
\begin{aligned}
& P(11-10)=P(8-7) \\
& =\left[\begin{array}{ccccccccccccccccc}
0.253 & 0.076 & 0.071 & 0.096 & 0.081 & 0.089 & 0.084 & 0.077 & 0.018 & 0.015 & 0.016 & 0.018 & 0.016 & 0.015 & 0.013 & 0.062 \\
0.075 & 0.329 & 0.089 & 0.084 & 0.082 & 0.082 & 0.090 & 0.081 & 0.008 & 0.012 & 0.009 & 0.013 & 0.010 & 0.008 & 0.009 & 0.018 \\
0.071 & 0.082 & 0.300 & 0.092 & 0.083 & 0.082 & 0.080 & 0.094 & 0.010 & 0.015 & 0.014 & 0.013 & 0.018 & 0.018 & 0.016 & 0.011 \\
0.075 & 0.074 & 0.075 & 0.351 & 0.085 & 0.073 & 0.082 & 0.081 & 0.013 & 0.009 & 0.014 & 0.016 & 0.010 & 0.016 & 0.013 & 0.014 \\
0.098 & 0.071 & 0.070 & 0.074 & 0.264 & 0.079 & 0.075 & 0.098 & 0.009 & 0.014 & 0.015 & 0.010 & 0.012 & 0.016 & 0.015 & 0.079 \\
0.089 & 0.087 & 0.080 & 0.080 & 0.083 & 0.306 & 0.095 & 0.093 & 0.014 & 0.011 & 0.010 & 0.009 & 0.012 & 0.010 & 0.013 & 0.009 \\
0.079 & 0.097 & 0.072 & 0.088 & 0.087 & 0.081 & 0.312 & 0.099 & 0.010 & 0.010 & 0.012 & 0.015 & 0.013 & 0.009 & 0.009 & 0.007 \\
0.095 & 0.096 & 0.090 & 0.081 & 0.091 & 0.090 & 0.085 & 0.302 & 0.012 & 0.010 & 0.008 & 0.008 & 0.007 & 0.008 & 0.010 & 0.005 \\
0.012 & 0.009 & 0.015 & 0.016 & 0.016 & 0.010 & 0.009 & 0.010 & 0.442 & 0.086 & 0.081 & 0.293 & 0 & 0 & 0 & 0 \\
0.009 & 0.015 & 0.008 & 0.011 & 0.016 & 0.015 & 0.011 & 0.010 & 0.081 & 0.342 & 0.077 & 0.405 & 0 & 0 & 0 & 0 \\
0.013 & 0.010 & 0.011 & 0.009 & 0.008 & 0.012 & 0.014 & 0.008 & 0.157 & 0.188 & 0.341 & 0.229 & 0 & 0 & 0 & 0 \\
0.015 & 0.016 & 0.015 & 0.016 & 0.014 & 0.019 & 0.017 & 0.033 & 0.272 & 0.294 & 0.289 & 0.522 & 0 & 0 & 0 & 0 \\
0.012 & 0.008 & 0.011 & 0.012 & 0.009 & 0.013 & 0.011 & 0.012 & 0 & 0 & 0 & 0 & 0.242 & 0.071 & 0.095 & 0.505 \\
0.014 & 0.011 & 0.016 & 0.015 & 0.016 & 0.009 & 0.015 & 0.016 & 0 & 0 & 0 & 0 & 0.098 & 0.320 & 0.094 & 0.375 \\
0.015 & 0.013 & 0.014 & 0.015 & 0.010 & 0.011 & 0.014 & 0.014 & 0 & 0 & 0 & 0 & 0.080 & 0.085 & 0.383 & 0.345 \\
0.016 & 0.013 & 0.015 & 0.015 & 0.010 & 0.015 & 0.014 & 0.011 & 0 & 0 & 0 & 0 & 0.105 & 0.157 & 0.135 & 0.495
\end{array}\right],
\end{aligned}
$$$$
P(5-4)
$$

$\left[\begin{array}{ccccccccccccccccc}0.311 & 0.062 & 0.059 & 0.019 & 0.013 & 0.003 & 0.006 & 0.001 & 0.202 & 0.002 & 0.079 & 0.006 & 0.171 & 0.012 & 0.046 & 0.008 \\ 0.068 & 0.331 & 0.022 & 0.124 & 0.01 & 0.125 & 0.009 & 0.007 & 0.006 & 0.107 & 0.093 & 0.077 & 0.012 & 0.001 & 0.007 & 0.001 \\ 0.092 & 0.131 & 0.430 & 0.103 & 0.03 & 0.012 & 0.008 & 0.012 & 0.003 & 0.001 & 0.005 & 0.002 & 0.011 & 0.038 & 0.012 & 0.110 \\ 0.095 & 0.124 & 0.118 & 0.485 & 0.011 & 0.009 & 0.008 & 0.111 & 0.001 & 0.009 & 0.001 & 0.003 & 0.007 & 0.010 & 0.003 & 0.005 \\ 0.072 & 0.007 & 0.004 & 0.006 & 0.407 & 0.085 & 0.094 & 0.051 & 0.002 & 0.007 & 0.086 & 0.082 & 0.001 & 0.077 & 0.006 & 0.013 \\ 0.001 & 0.071 & 0.007 & 0.015 & 0.081 & 0.377 & 0.127 & 0.083 & 0.001 & 0.034 & 0.110 & 0.073 & 0.003 & 0.001 & 0.014 & 0.002 \\ 0.006 & 0.001 & 0.072 & 0.013 & 0.075 & 0.092 & 0.490 & 0.110 & 0.008 & 0.003 & 0.010 & 0.001 & 0.087 & 0.094 & 0.016 & 0.021 \\ 0.005 & 0.005 & 0.018 & 0.043 & 0.114 & 0.201 & 0.102 & 0.467 & 0.010 & 0.002 & 0.004 & 0.012 & 0.001 & 0.003 & 0.011 & 0.002 \\ 0.111 & 0.065 & 0.008 & 0.024 & 0.014 & 0.005 & 0.011 & 0.04 & 0.462 & 0.054 & 0.124 & 0.082 & 0 & 0 & 0 & 0 \\ 0.001 & 0.003 & 0.070 & 0.010 & 0.002 & 0.006 & 0.004 & 0.021 & 0.104 & 0.469 & 0.128 & 0.182 & 0 & 0 & 0 & 0 \\ 0.011 & 0.032 & 0.044 & 0.012 & 0.011 & 0.003 & 0.013 & 0.06 & 0.101 & 0.078 & 0.521 & 0.144 & 0 & 0 & 0 & 0 \\ 0.002 & 0.012 & 0.001 & 0.002 & 0.011 & 0.005 & 0.012 & 0.004 & 0.174 & 0.097 & 0.124 & 0.556 & 0 & 0 & 0 & 0 \\ 0.089 & 0.029 & 0.002 & 0.004 & 0.001 & 0.078 & 0.046 & 0.047 & 0 & 0 & 0 & 0 & 0.360 & 0.121 & 0.134 & 0.089 \\ 0.011 & 0.003 & 0.001 & 0.001 & 0.009 & 0.006 & 0.002 & 0.004 & 0 & 0 & 0 & 0 & 0.171 & 0.449 & 0.166 & 0.177 \\ 0.005 & 0.049 & 0.003 & 0.026 & 0.120 & 0.001 & 0.133 & 0.003 & 0 & 0 & 0 & 0 & 0.123 & 0.125 & 0.306 & 0.106 \\ 0.002 & 0.001 & 0.004 & 0.007 & 0.011 & 0.004 & 0.008 & 0.002 & 0 & 0 & 0 & 0 & 0.119 & 0.126 & 0.175 & 0.541\end{array}\right]$




$$
\begin{aligned}
& P(6-5)=P(9-8)=P(12-11)=P(2-1) \\
& {\left[\begin{array}{ccccccccccccccccccc}
0.207 & 0.021 & 0.065 & 0.019 & 0.021 & 0.001 & 0.002 & 0.002 & 0.212 & 0.005 & 0.163 & 0.002 & 0.179 & 0.021 & 0.067 & 0.013 \\
0.022 & 0.201 & 0.031 & 0.213 & 0.014 & 0.129 & 0.006 & 0.001 & 0.006 & 0.191 & 0.163 & 0.013 & 0.001 & 0.002 & 0.001 & 0.006 \\
0.029 & 0.063 & 0.52 & 0.151 & 0.001 & 0.016 & 0.007 & 0.002 & 0.005 & 0.001 & 0.003 & 0.004 & 0.012 & 0.051 & 0.014 & 0.121 \\
0.028 & 0.057 & 0.132 & 0.684 & 0.015 & 0.007 & 0.004 & 0.019 & 0.011 & 0.009 & 0.007 & 0.013 & 0.006 & 0.004 & 0.001 & 0.003 \\
0.024 & 0.002 & 0.005 & 0.009 & 0.552 & 0.072 & 0.053 & 0.008 & 0.002 & 0.006 & 0.123 & 0.012 & 0.012 & 0.09 & 0.021 & 0.009 \\
0.001 & 0.044 & 0.01 & 0.021 & 0.119 & 0.421 & 0.084 & 0.017 & 0.003 & 0.058 & 0.192 & 0.013 & 0.002 & 0.001 & 0.005 & 0.009 \\
0.002 & 0.005 & 0.106 & 0.026 & 0.132 & 0.099 & 0.340 & 0.002 & 0.006 & 0.001 & 0.003 & 0.011 & 0.079 & 0.14 & 0.02 & 0.028 \\
0.003 & 0.004 & 0.037 & 0.111 & 0.251 & 0.302 & 0.102 & 0.155 & 0.002 & 0.001 & 0.010 & 0.003 & 0.001 & 0.006 & 0.011 & 0.001 \\
0.042 & 0.039 & 0.103 & 0.044 & 0.025 & 0.005 & 0.008 & 0.009 & 0.047 & 0.103 & 0.227 & 0.015 & 0 & 0 & 0 & 0 \\
0.012 & 0.010 & 0.071 & 0.005 & 0.002 & 0.001 & 0.009 & 0.006 & 0.072 & 0.648 & 0.161 & 0.023 & 0 & 0 & 0 & 0 \\
0.003 & 0.017 & 0.04 & 0.016 & 0.014 & 0.002 & 0.007 & 0.009 & 0.076 & 0.108 & 0.693 & 0.015 & 0 & 0 & 0 & 0 \\
0.001 & 0.01 & 0.008 & 0.005 & 0.027 & 0.001 & 0.003 & 0.007 & 0.234 & 0.251 & 0.306 & 0.146 & 0 & 0 & 0 & 0 \\
0.034 & 0.017 & 0.003 & 0.012 & 0.001 & 0.086 & 0.033 & 0.01 & 0 & 0 & 0 & 0 & 0.326 & 0.183 & 0.173 & 0.122 \\
0.003 & 0.001 & 0.012 & 0.003 & 0.001 & 0.015 & 0.010 & 0.017 & 0 & 0 & 0 & 0 & 0.112 & 0.494 & 0.155 & 0.177 \\
0.001 & 0.024 & 0.004 & 0.039 & 0.171 & 0.002 & 0.076 & 0.001 & 0 & 0 & 0 & 0 & 0.091 & 0.16 & 0.313 & 0.118 \\
0.003 & 0.002 & 0.015 & 0.006 & 0.011 & 0.002 & 0.008 & 0.009 & 0 & 0 & 0 & 0 & 0.084 & 0.164 & 0.175 & 0.538
\end{array}\right],} \\
& P(4-3)
\end{aligned}
$$$$
=\left[\begin{array}{cccccccccccccccccc}
0.278 & 0.096 & 0.087 & 0.056 & 0.078 & 0.076 & 0.087 & 0.069 & 0.036 & 0.027 & 0.030 & 0.029 & 0.015 & 0.010 & 0.010 & 0.012 \\
0.087 & 0.322 & 0.079 & 0.085 & 0.084 & 0.090 & 0.071 & 0.084 & 0.012 & 0.007 & 0.011 & 0.011 & 0.009 & 0.018 & 0.017 & 0.012 \\
0.079 & 0.078 & 0.307 & 0.086 & 0.088 & 0.091 & 0.071 & 0.084 & 0.018 & 0.015 & 0.014 & 0.019 & 0.017 & 0.012 & 0.009 & 0.013 \\
0.089 & 0.074 & 0.076 & 0.401 & 0.090 & 0.070 & 0.075 & 0.012 & 0.014 & 0.013 & 0.016 & 0.014 & 0.011 & 0.011 & 0.010 & 0.023 \\
0.007 & 0.002 & 0.051 & 0.085 & 0.437 & 0.116 & 0.143 & 0.080 & 0.005 & 0.011 & 0.010 & 0.010 & 0.014 & 0.012 & 0.001 & 0.015 \\
0.100 & 0.078 & 0.099 & 0.075 & 0.086 & 0.311 & 0.080 & 0.082 & 0.011 & 0.010 & 0.009 & 0.010 & 0.013 & 0.010 & 0.013 & 0.014 \\
0.082 & 0.080 & 0.089 & 0.083 & 0.087 & 0.072 & 0.336 & 0.079 & 0.010 & 0.018 & 0.007 & 0.013 & 0.009 & 0.016 & 0.011 & 0.008 \\
0.090 & 0.080 & 0.083 & 0.074 & 0.089 & 0.071 & 0.076 & 0.303 & 0.020 & 0.012 & 0.020 & 0.017 & 0.017 & 0.011 & 0.018 & 0.021 \\
0.021 & 0.011 & 0.012 & 0.012 & 0.018 & 0.013 & 0.021 & 0.014 & 0.419 & 0.194 & 0.137 & 0.128 & 0 & 0 & 0 & 0 \\
0.012 & 0.017 & 0.020 & 0.018 & 0.016 & 0.017 & 0.019 & 0.011 & 0.075 & 0.398 & 0.225 & 0.172 & 0 & 0 & 0 & 0 \\
0.020 & 0.014 & 0.017 & 0.015 & 0.018 & 0.020 & 0.018 & 0.012 & 0.184 & 0.181 & 0.362 & 0.139 & 0 & 0 & 0 & 0 \\
0.011 & 0.022 & 0.013 & 0.020 & 0.016 & 0.018 & 0.015 & 0.012 & 0.169 & 0.129 & 0.128 & 0.447 & 0 & 0 & 0 & 0 \\
0.025 & 0.021 & 0.029 & 0.021 & 0.020 & 0.024 & 0.028 & 0.026 & 0 & 0 & 0 & 0 & 0.282 & 0.101 & 0.186 & 0.238 \\
0.013 & 0.011 & 0.013 & 0.021 & 0.017 & 0.016 & 0.021 & 0.015 & 0 & 0 & 0 & 0 & 0.146 & 0.351 & 0.183 & 0.192 \\
0.016 & 0.020 & 0.017 & 0.021 & 0.014 & 0.020 & 0.014 & 0.013 & 0 & 0 & 0 & 0 & 0.186 & 0.173 & 0.396 & 0.108 \\
0.011 & 0.020 & 0.013 & 0.016 & 0.019 & 0.011 & 0.016 & 0.013 & 0 & 0 & 0 & 0 & 0.156 & 0.087 & 0.147 & 0.492
\end{array}\right] .
$$ 
TABLE 1: Verification results of actual driving experiment.

\begin{tabular}{|c|c|c|c|c|}
\hline \multirow{2}{*}{ Driver number } & \multirow{2}{*}{ Reconstruction times } & \multicolumn{2}{|c|}{ Comparison of reconstruction results with actual situation } & \multirow{2}{*}{ Accuracy rate } \\
\hline & & Match times & No match times & \\
\hline 2 & 50 & 42 & 8 & $84 \%$ \\
\hline 5 & 50 & 39 & 11 & $78 \%$ \\
\hline 6 & 50 & 40 & 10 & $80 \%$ \\
\hline 9 & 50 & 41 & 9 & $82 \%$ \\
\hline 13 & 50 & 39 & 11 & $78 \%$ \\
\hline 16 & 50 & 40 & 10 & $80 \%$ \\
\hline 17 & 50 & 39 & 11 & $78 \%$ \\
\hline 19 & 50 & 42 & 8 & $84 \%$ \\
\hline 22 & 50 & 37 & 13 & $74 \%$ \\
\hline 25 & 50 & 41 & 9 & $82 \%$ \\
\hline 26 & 50 & 39 & 11 & $78 \%$ \\
\hline 30 & 50 & 40 & 10 & $80 \%$ \\
\hline 32 & 50 & 42 & 8 & $84 \%$ \\
\hline 33 & 50 & 37 & 13 & $74 \%$ \\
\hline 37 & 50 & 42 & 8 & $84 \%$ \\
\hline 40 & 50 & 40 & 10 & $80 \%$ \\
\hline 42 & 50 & 38 & 12 & $76 \%$ \\
\hline 45 & 50 & 41 & 9 & $82 \%$ \\
\hline 47 & 50 & 38 & 12 & $76 \%$ \\
\hline 50 & 50 & 39 & 11 & $78 \%$ \\
\hline
\end{tabular}

Similarly, for midsize vehicle and large vehicle, the transition matrix of the vehicle group situation in some regions also could be obtained according to the proposed method. Due to the limited space, these specific analyses are no longer demonstrated here.

Based on the identification of the vehicle group situation which was constituted by the target vehicle and its neighboring vehicles when the target vehicle arrived at VTL2, VTL4, and VTL6, the vehicle group situation in the road network would be reconstructed according to the corresponding transition matrix of each region.

\subsection{Discussion}

5.2.1. Actual Driving Verification. Partial experimental data of 20 drivers which was not used for the model calibration was selected to verify the model. 50 groups of data were selected to reconstruct the vehicle group situation. In other words, 50 transitions of vehicle group situation for each driver were reconstructed. The reconstruction results were compared with the realities of vehicle group situation. The calculation with a penetration rate of $20 \%$ was selected to illustrate reconstruction accuracy of the calculation method. The results of actual driving experiment were shown as in Table 1.

It can be concluded from Table 1 that the average accuracy rate was $79.6 \%$ when the penetration rate was $20 \%$. The average accuracy rate (abbreviated as accuracy rate hereinafter) was used to evaluate the accuracy. The accuracy rate with

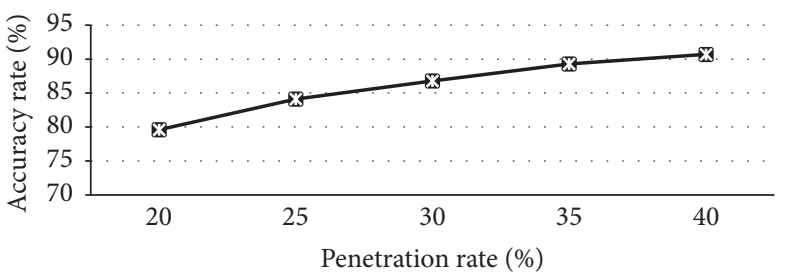

FIGURE 11: Accuracy rate of vehicle group situation reconstruction.

the penetration rate of $20 \%, 25 \%, 30 \%, 35 \%$, and $40 \%$ was calculated. The accuracy rate with different penetration rates was shown in Figure 11.

It can be concluded from Figure 11 that the accuracy rate of the model was about $80 \%$ when the penetration rate was $20 \%$. With the increase of the penetration rate, the accuracy rate increased continuously. The magnitude of the reconstructed accuracy rate was higher when the penetration rate increased from $20 \%$ to $30 \%$. The increase in amplitude of accuracy rate was relatively small when the penetration rate exceeded $30 \%$. Overall, the accuracy rate of vehicle group situation reconstructed method proposed in this paper was high, and the method was reasonable and feasible.

5.2.2. Virtual Driving Verification. Parallel interactive driving simulation experiment on driving simulators was designed to validate the model as shown in Figure 12.

The penetration rate of $20 \%$ was taken as an example, and the virtual driving experiment results were shown in Table 2. 
TABLE 2: Results of virtual driving experiment.

\begin{tabular}{|c|c|c|c|c|}
\hline \multirow{2}{*}{ Driver number } & \multirow{2}{*}{ Reconstruction times } & \multicolumn{2}{|c|}{ Comparison of reconstruction results with actual situation } & \multirow{2}{*}{ Accuracy rate } \\
\hline & & Match times & No match times & \\
\hline 1 & 50 & 41 & 9 & $82 \%$ \\
\hline 2 & 50 & 38 & 12 & $76 \%$ \\
\hline 3 & 50 & 41 & 9 & $82 \%$ \\
\hline 4 & 50 & 39 & 11 & $78 \%$ \\
\hline 5 & 50 & 40 & 10 & $80 \%$ \\
\hline 6 & 50 & 40 & 10 & $80 \%$ \\
\hline 7 & 50 & 39 & 11 & $78 \%$ \\
\hline 8 & 50 & 41 & 9 & $82 \%$ \\
\hline 9 & 50 & 38 & 12 & $76 \%$ \\
\hline 10 & 50 & 40 & 10 & $80 \%$ \\
\hline
\end{tabular}
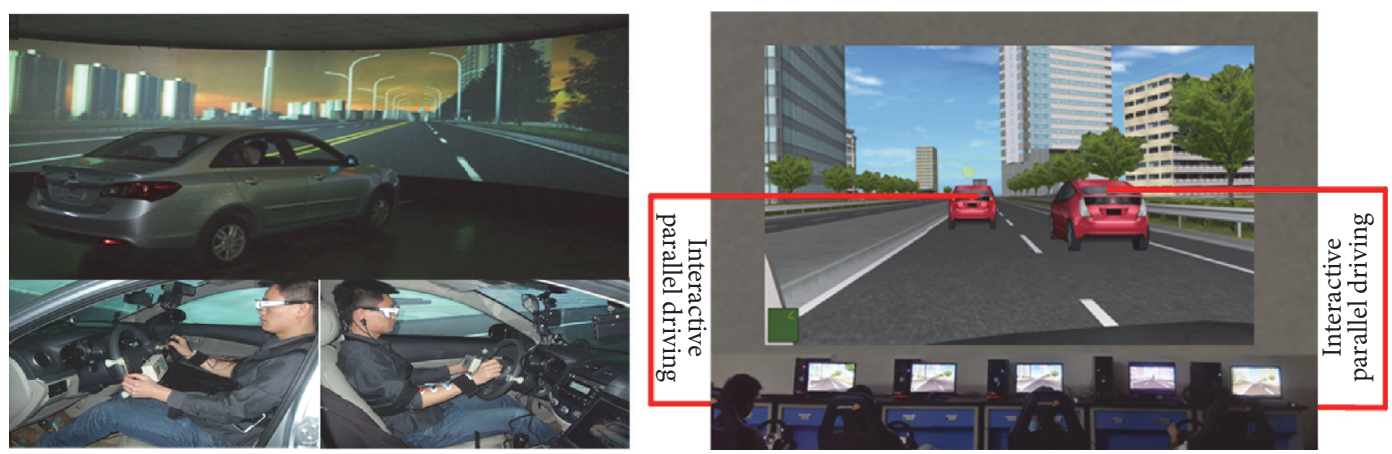

Figure 12: Virtual driving experiment.

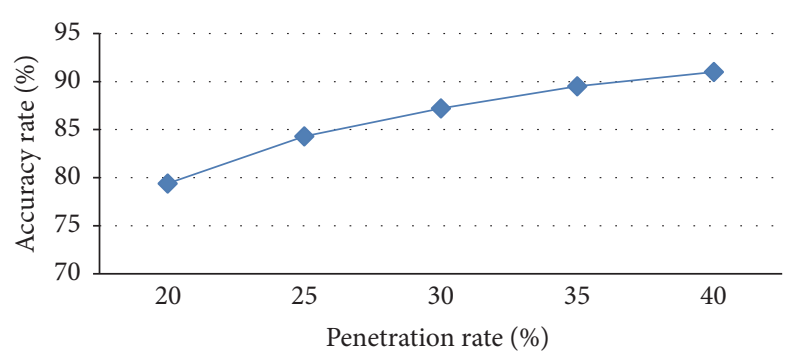

FIGURE 13: Accuracy rate of vehicle group situation reconstruction.

With the same method, the accuracy of the corresponding method under different penetration rates was shown in Figure 13.

As can be seen from Table 2 and Figure 13, the vehicle group situation reconstruction results obtained through the proposed method in the virtual driving experiment had a high reliability. The accuracy of the method was about $79 \%$ when the penetration rate was $20 \%$. The accuracy rate was above $80 \%$ when the penetration rate was $25 \%$. And the accuracy rate would increase with the increase of the penetration rate.

5.2.3. Simulation Verification. The driving situation of target vehicle was taken as an example, and the simulation results were shown in Figures 14 and 15, where the actual value was the difference between the measured data of the front and

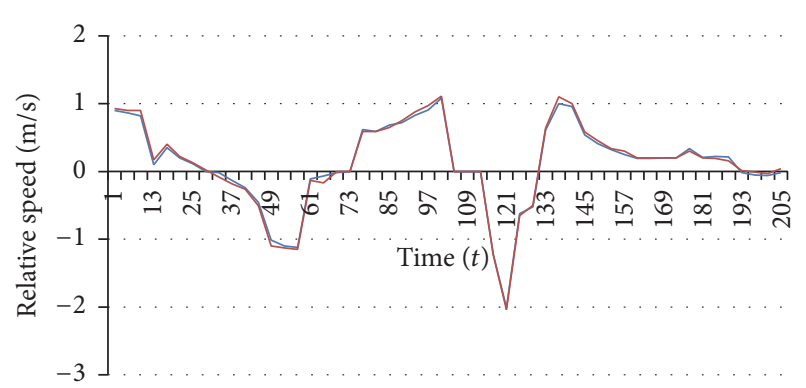

- Actual value

- Simulation value

FIGURE 14: Relative speed simulation verification.

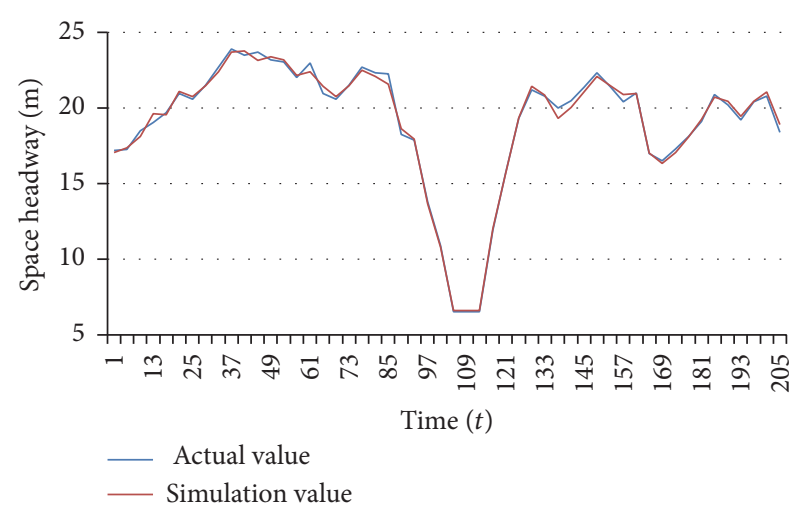

FIGURE 15: Space headway simulation verification. 
rear vehicle (the target vehicle was the rear vehicle), and the simulated value was the difference between the rear vehicle (the target vehicle was the rear vehicle) simulation data which considered the time-varying law of vehicle group situation during simulation process and the front vehicle measured data.

The verification results showed that the microscopic simulation which considered time-varying law of vehicle group situation based on driver-vehicle feature evolution had good consistency with the actual situation, and it could accurately simulate the behavior of the driver.

The verification results of actual driving, virtual driving, and simulation showed that the proposed method was scientific and reasonable.

\section{Conclusion}

In this paper, the factors that affect the vehicle condition and the driving propensity during driving were comprehensively considered. And the three-lane scene was taken as an example, and the reverse reconstruction method of vehicle group situation in urban road network was proposed. Finally, the experiments of actual driving, virtual driving, and simulation were designed to verify the method. The results showed that the proposed method had a higher accuracy. The accuracy rate of the method reached $79 \%$ when the penetration rate of experimental vehicle was $20 \%$, and the accuracy of the method would increase with the increase of penetration rate. Overall, the method was scientific and reasonable. It is worth mentioning that the accuracy of the method proposed in this paper was high even with the limited information of vehicles and drivers. The method also adapt to the situations when the data quantity was very large, the research area was large, and the data collection was difficult in the study area, and the time and cost could be saved to a certain extent.

\section{Competing Interests}

The authors declare that they have no competing interests.

\section{Acknowledgments}

This study was supported by the State Key Laboratory of Automotive Safety and Energy under Project no. KF16232, Natural Science Foundation of Shandong Province (Grant nos. ZR2014FM027, ZR2016EL19), Social Science Planning Project of Shandong Province (Grant no. 14CGLJ27), Project of Shandong Province Higher Educational Science and Technology Program (Grant no. J15LB07), and the National Natural Science Foundation of China (Grant nos. 61074140, 61573009, 1508315, and 51608313).

\section{References}

[1] X. Wang, J. Liu, and J. Zhang, "Dynamic recognition model of driver's propensity under multilane traffic environments," Discrete Dynamics in Nature and Society, vol. 2012, Article ID 309415, 15 pages, 2012.
2] E. Käfer, C. Hermes, C. Wöhler, F. Kummert, and H. Ritter, "Recognition and prediction of situations in urban traffic scenarios," in Proceedings of the 20th International Conference on Pattern Recognition (ICPR '10), pp. 4234-4237, IEEE, Istanbul, Turkey, August 2010.

[3] D. Meyer-Delius, C. Plagemann, and W. Burgard, "Probabilistic situation recognition for vehicular traffic scenarios," in Proceedings of the IEEE International Conference on Robotics and Automation (ICRA '09), pp. 459-464, IEEE, Kobe, Japan, May 2009.

[4] J. Zhang, X. Wang, J. Wang, and X. Ban, "Vehicle group relationship transformation mechanism under dynamic and complex three-lane conditions," Advances in Mechanical Engineering, vol. 7, no. 2, Article ID 687517, 2015.

[5] Z. Sun and J. Ban, "Vehicle trajectory reconstruction for signalized intersections using mobile traffic sensors," Transportation Research C, vol. 36, pp. 268-283, 2013, Proceedings of the 91st Transportation Research Board Annual Meeting, 2012.

[6] X. Ban and M. Gruteser, "Towards fine-grained urban traffic knowledge extraction using mobile sensing," in Proceedings of the International Workshop on Urban Computing (UrbComp '12)-Held in Conjunctionwith (KDD '12), pp. 111-117, Beijing, China, August 2012.

[7] X. Wang, J. Wang, J. Zhang, and J. Wang, "Dynamic recognition of driver's propensity based on GPS mobile sensing data and privacy protection," Mathematical Problems in Engineering, vol. 2016, Article ID 1814608, 12 pages, 2016.

[8] X. Wang, J. Zhang, and L. Ma, "Estimation of gamma distribution shape parameter adapting to traffic flow evolvement," Computer Engineering \& Applications, vol. 50, no. 5, pp. 247251, 2014.

[9] Y. Jiang, S. Han, W. Ting, and G. Zhang, "Analysis of vehicle arrival law at signalized intersection," Journal of Transportation Engineering \& Information, vol. 13, no. 1, pp. 1-6, 2015.

[10] M. Li, H. Lu, and C. Nie, "Urban road capacity algorithm based on multi-variant gamma distribution," Highway Engineering, vol. 34, no. 4, pp. 136-140, 2009.

[11] J. Zhang, X. Wang, C. Yu, Z. Liu, and H. Wang, "Development of a prediction method for driver's propensity," Procedia Engineering, vol. 137, pp. 161-170, 2016.

[12] M. Wistrand and E. L. L. Sonnhammer, "Improving profile HMM discrimination by adapting transition probabilities," Journal of Molecular Biology, vol. 338, no. 4, pp. 847-854, 2004.

[13] J. C. Herrera, D. B. Work, R. Herring, X. Ban, Q. Jacobson, and A. M. Bayen, "Evaluation of traffic data obtained via GPSenabled mobile phones: the mobile century, field experiment," Transportation Research Part C Emerging Technologies, vol. 18, no. 4, pp. 568-583, 2009.

[14] M. M. Al-Sayed, S. Khattab, and F. A. Omara, "Prediction mechanisms for monitoring state of cloud resources using Markov chain model," Journal of Parallel and Distributed Computing, vol. 96, pp. 163-171, 2016.

[15] G. A. Pavliotis and A. M. Stuart, Multiscale methods, vol. 53 of Texts in Applied Mathematics, Springer, New York, NY, USA, 2008 


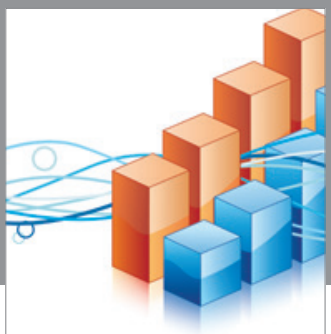

Advances in

Operations Research

vatem alat4

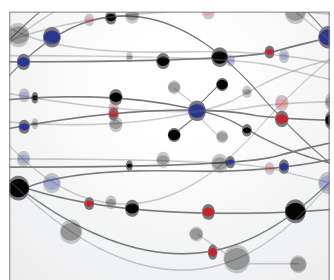

\section{The Scientific} World Journal
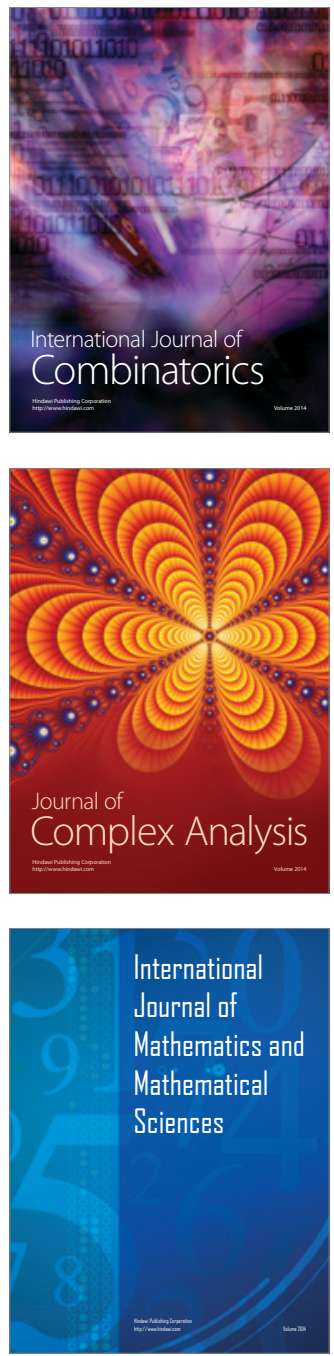
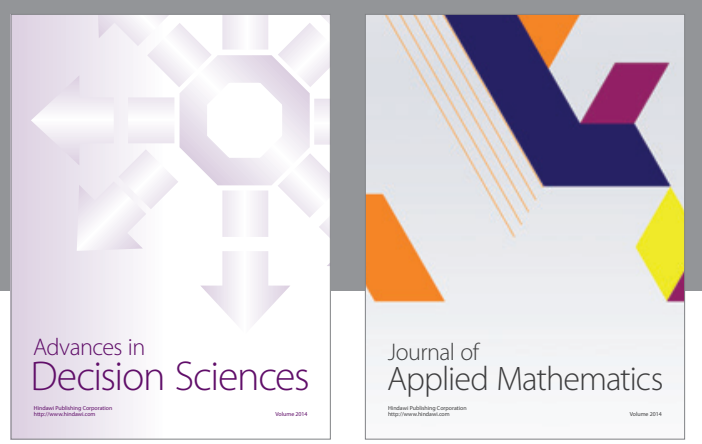

Algebra

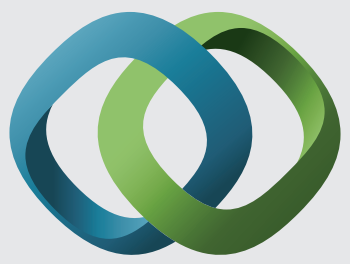

\section{Hindawi}

Submit your manuscripts at

https://www.hindawi.com
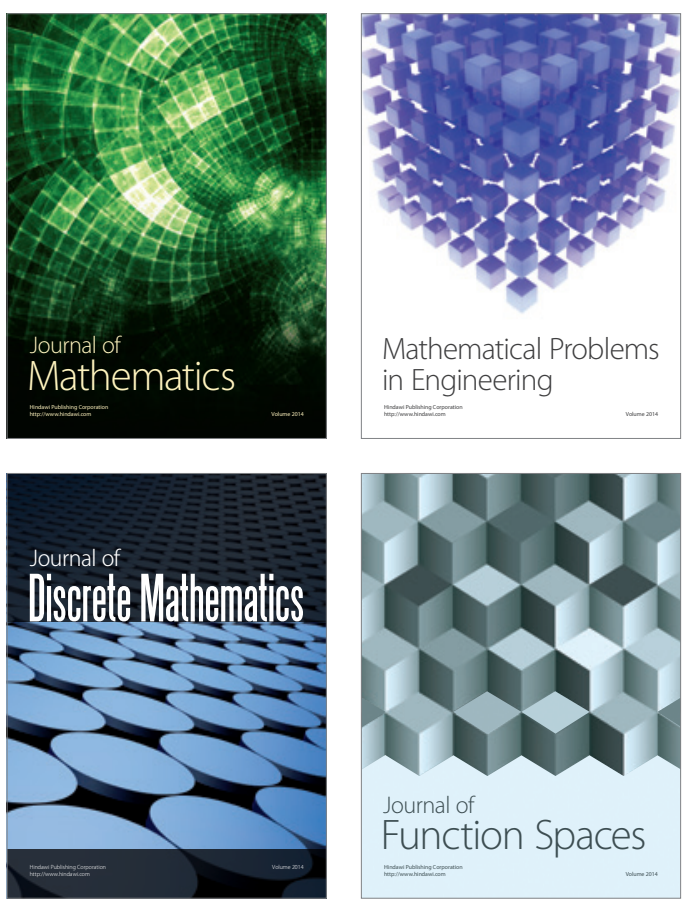

Mathematical Problems in Engineering
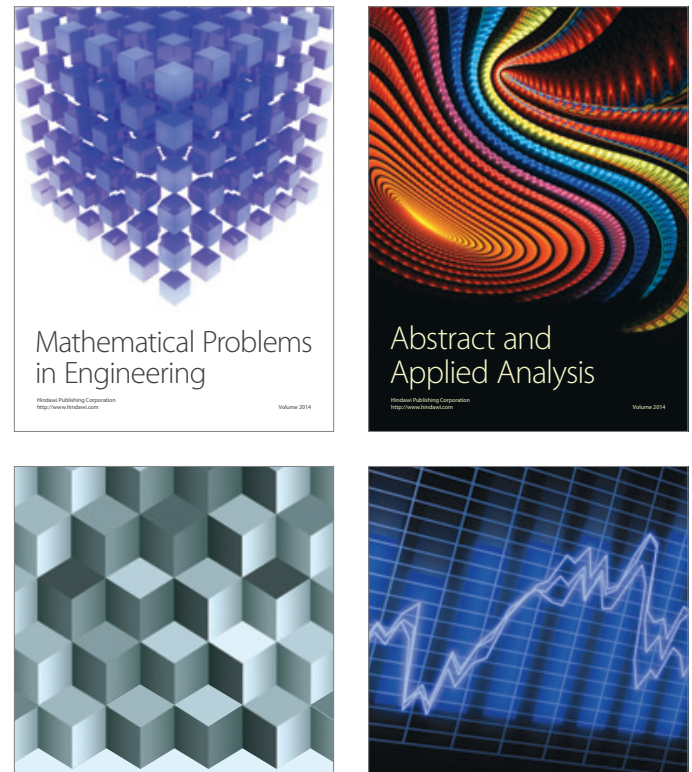

Journal of

Function Spaces

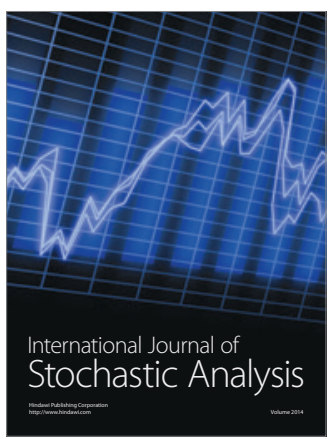

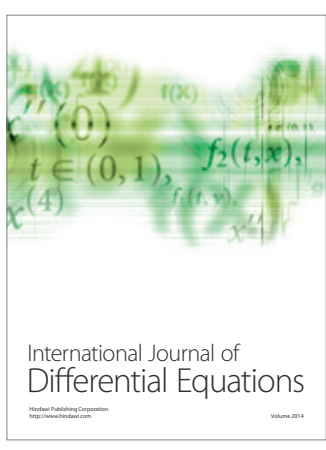
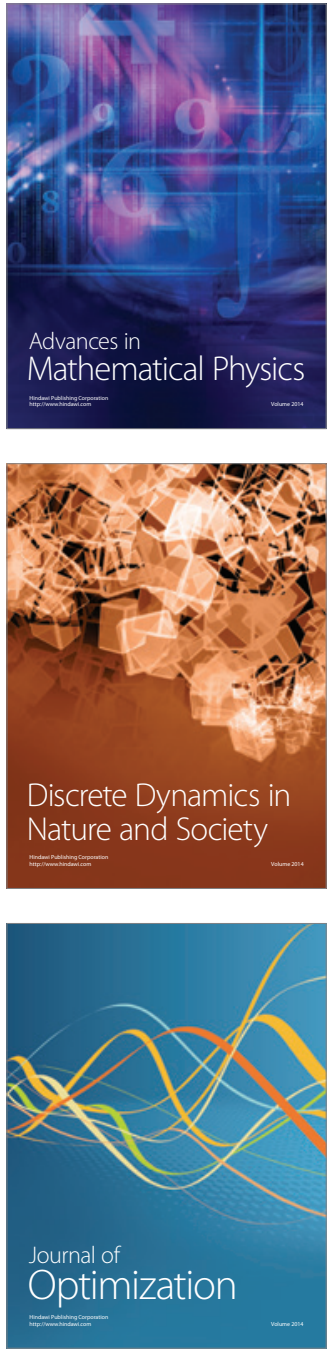